\title{
Norepinephrine reduces Arterial Compliance less than Phenylephrine when Treating General Anesthesia-induced Arterial Hypotension.
}

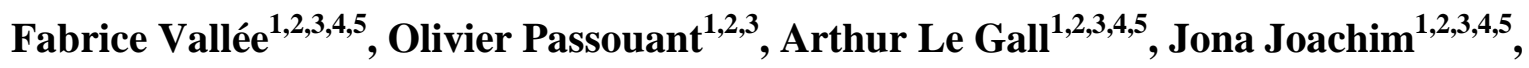 \\ Joaquim Mateo $^{1,2,3}$, Alexandre Mebazaa ${ }^{1,2,3}$, Etienne Gayat ${ }^{1,2,3}$ \\ ${ }^{1}$ Département d'Anesthésie - Réanimation - SMUR, Hôpitaux Universitaires Saint Louis - Lariboisière, Assistance \\ Publique - Hôpitaux de Paris, Paris, France. ${ }^{2}$ Université Paris Diderot, Paris, France. ${ }^{3}$ UMR-S 942, INSERM, Paris, \\ France. Hôpitaux Universitaires Saint Louis - Lariboisière, Assistance Publique - Hôpitaux de Paris, Paris, France. ${ }^{4}$ \\ LMS, Ecole Polytechnique, CNRS, Université Paris-Saclay. ${ }^{5}$ M3DISIM, Inria, Université Paris-Saclay.
}

Short title: Norepinephrine compared to phenylephrine

Word count (excl. abstract and references): 2570

IRB Information: Institutional Review Board of the Société de Réanimation de Langue Française (CE SRLF 11-356).

Solely the institution provided support.

$\underline{\text { No Conflict of Interest }}$

Corresponding author: Dr Fabrice Vallée Department of Anesthesiology and Critical Care Hôpitaux Universitaires Saint-Louis-Lariboisière Assistance Publique - Hôpitaux de Paris Université Paris Diderot 2 rue Ambroise-Paris 75475 PARIS Cedex 10, France.

Phone: +33-149958071 Fax: +33-149958073

E-mail address: vallee.fabrice@gmail.com 


\begin{abstract}
Introduction: During general anesthesia, arterial hypotension is frequent and may be an important contributor to peri-operative morbidity. We assessed the effect of a $5 \mu \mathrm{g}$ bolus of Norepinephrine (NA) when compared with $50 \mu \mathrm{g}$ bolus of Phenylephrine (PE) administered to treat hypotension during maintenance anesthesia, on MAP, derived cardiac output and arterial stiffness parameters. Methods: Patients scheduled for a neurosurgical procedure under general anesthesia were prospectively included. Monitoring included invasive blood pressure, esophageal Doppler and arterial tonometer used to estimate central aortic pressure with arterial stiffness parameters, such as augmentation index (Aix). After initial resuscitation, hypotensive episodes were corrected by a bolus administration of NA or PE in a peripheral venous line. Results: There were 269 bolus administrations of vasopressors (149 NA, $120 \mathrm{PE}$ ) in 47 patients with no adverse effects detected. A decrease in stroke volume (SV) was observed with PE compared with NA ($18 \pm 9 \%$ vs $-14 \pm 7 \%, \mathrm{p}<0.001)$. This decrease was associated with an increase in Aix, which was greater for PE than for NA $(+10 \pm 8 \%$ vs $+6 \pm 6 \%, \mathrm{p}<0.0001)$, and a decrease in total arterial compliance greater for PE compared to NA (Ctot=SV/Central Pulse Pressure) (-35 $\pm 9 \%$ vs. $29 \pm 10 \%, \mathrm{p}<0.001)$. Discussion: This study suggests that $5 \mu \mathrm{g}$ of NA administered as a bolus in a peripheral venous line could treat general-anesthesia-induced arterial hypotension with a smaller decrease in SV and arterial compliance when compared to PE.
\end{abstract}

Key-words: vasopressors, general anesthesia, hypotension, ventricular arterial coupling, Doppler, tonometry 


\section{Introduction}

During general anesthesia, arterial hypotension is frequent and may be an important contributor to peri-operative morbidity ${ }^{1-3}$. Hypotension has multiple and intricate underlying mechanisms: vasoplegia due to sympatholysis ${ }^{4}$ and to a decrease in circulating catecholamines ${ }^{5}$ induced by anesthetic agents, hypovolemia and myocardial dysfunction. Current strategies for restoring perfusion pressure during general anesthesia are based on volume expansion and/or intravascular administration of vasopressors ${ }^{6}$. Vasopressive agents often combine vasoconstriction and direct or indirect myocardial effects, depending on the drug used ${ }^{7}$. In daily practice, ephedrine (EPH) is a very commonly used vasopressor. However, it can induce tachycardia and tachyphylaxis, and therefore, its cumulative dose is limited. Phenylephrine (PE) is widely used during anesthesia often as a relay of EPH - but this "standard of care", validated by practice, has been recently challenged. ${ }^{8,9}$ Indeed, documented physiological effects on cardiac output and left ventricular afterload may be detrimental, while some regional hemodynamic advantages remain advocated by some authors in particular settings (as critical aortic stenosis, decompensated tetralogy of Fallot, and hypotension during caesarean delivery). Moreover, PE may be responsible for bradycardia via baroreflex activation ${ }^{9}$ and alteration of left ventricular function ${ }^{10,11}$. As recently suggested $^{12}$, norepinephrine (NA) may represent a valuable alternative to PE to treat general anesthesia-induced hypotension. Indeed, Wuethrich et al showed that the intra-operative continuous infusion of low-dose NA in high-risk surgical patients could limit fluid infusion during major surgery ${ }^{13,14}$. Moreover, few studies suggest a beneficial effect of NA compared to PE to control hypotension induced by spinal anesthesia in patients having cesarean delivery ${ }^{15}$ and in patients with chronic pulmonary hypertension ${ }^{16}$. However, the question of the superiority of NA compared to PE to restore mean arterial pressure in daily clinical practice is still a matter of 
debate with some controversial results. Indeed, a recent randomized controlled trial confirmed the possibilities to treat general anesthesia induced arterial hypotension with low dose of NA, but failed to show statistical difference between NA and PE in term of cardiac output, heart rate and tissue perfusion variations ${ }^{17}$. To our knowledge, none of those studies assessed the differential effect of those drugs on arterial stiffness or on ventricular-arterial coupling.

Along with the expected increase in mean arterial pressure resulting from vasoconstriction, intravenous vasopressors may increase arterial stiffness, increase myocardial work and potentially decrease stroke volume and arterial compliance. These alterations in left ventricular arterial coupling could be associated with adverse outcomes ${ }^{18-20}$. Recently, noninvasive methods to quantify arterial stiffness have been developed. In particular, arterial tonometry has been used in many settings ${ }^{21}$, and many parameters have been derived to evaluate clinical repercussion of arterial stiffness ${ }^{22,23}$. The most commonly used parameters are augmentation pressure (AP) and augmentation index (Aix), which are measured after reconstruction of central wave pressure ${ }^{22}$ (Fig 1 of ESM). Many studies reported the detrimental effect of advanced age ${ }^{24,25}$, gender ${ }^{25-27}$, cardiovascular risk factors and hypotensive drugs on arterial stiffness using such methods, and demonstrated the necessity of studying the central pressure rather than the peripheral pressure in this indication ${ }^{20,28}$. However, very few studies have analyzed the effect of intra-operative vasopressive agents on cardiac performance and arterial stiffness.

The goal of this study was to compare effects of bolus of PE to low-dose NA administrated from a peripheral venous access on cardiac output and arterial stiffness when they are used to treat general-anesthesia-induced arterial hypotension. Effects of vasopressors were compared in the presence of absence of cardiovascular risk factors.

\section{Materials and Methods}




\section{Patients}

This prospective observational study included neurosurgical patients who were sedated under general anesthesia operated in supine position between February and July 2013 at Lariboisiere University Hospital (Paris, France). This study was approved by the Institutional Review Board of the Société de Réanimation de Langue Française (CE SRLF 11-356). Exclusion criteria were age $<18$ years, pregnancy and contraindication to the use of esophageal Doppler. Patients were assigned to one of two groups according to the number of cardiovascular risk factors they presented with. One group included patients who presented with 1 or no cardiovascular risk factor and was called the "Low CV risk" group, whereas the other group, called the "High CV risk" group, included patients who presented with at least 2 cardiovascular risk factors. Age $>50$ years, treated arterial hypertension, current smoking, diabetes mellitus, dyslipidemia were considered as cardiovascular risk factors. A history of cardiovascular events or congestive heart failure directly classified in "High CV risk group".

\section{Patient hemodynamic monitoring}

In our center, neurosurgical patients are monitored hemodynamically, combining invasive arterial pressure via a radial or femoral artery catheter, and cardiac output by trans-esophageal Doppler (EDCO). We used a device that allowed simultaneous recording of both invasive arterial pressure and cardiac output (CombiQ®, Deltex, Chichester, UK).

In parallel, aortic arterial pressure was estimated by arterial tonometry (Sphygmocor®, Atcor Medical). This device is composed of a micro manometer-tipped probe that can be applied against radial arterial wall with sufficient pressure to plane the artery. This application creates a signal that approximates instantaneous transmural pressure. After initial calibration of the tonometer with invasive blood pressure values, a validated mathematical transfer function 
provides an estimation of the central aortic pulse wave based on the radial signal ${ }^{29}$. In the present study, the probe was applied on the controlateral radial artery of the invasive arterial line, allowing repeated measurements with high stability and reproducibilityas already described ${ }^{30}$.

\section{Collected and calculated parameters}

- Systolic (SAP), diastolic (DAP) and mean (MAP) from the invasive arterial blood pressure.

- Stroke volume (SV) and cardiac output (CO) from the esophageal Doppler.

- Central pulse pressure (centralPP), augmentation pressure (AP) and augmentation index (Aix) from radial arterial tonometer (Fig 1 of ESM)

- Total arterial compliance $\mathrm{Ctot}=\mathrm{SV} /{ }_{\text {central }} \mathrm{PP}$, were calculated.

All these parameters were collected before the administration of vasopressors and at the peak effect on MAP.

\section{Study protocol}

All subjects were orally premedicated with hydroxyzine $\left(1 \mathrm{mg} \cdot \mathrm{kg}^{-1}\right) 1 \mathrm{~h}$ before surgery. Anesthesia was inducted with propofol and remifentanil target-controlled infusion. Tracheal intubation was facilitated with atracurium $0.5 \mathrm{mg} \cdot \mathrm{kg}^{-1}$, and mechanical ventilation with volumecontrolled mode was used with tidal volumes of $7 \mathrm{ml} / \mathrm{kg}^{-1}$, a positive end-expiratory pressure of 5 $\mathrm{cmH}_{2} \mathrm{O}$ and a respiratory rate of $12-16$ breaths $\min ^{-1}$ to maintain an end-tidal $\mathrm{CO}_{2}$ of approximately 35 to $38 \mathrm{~mm} \mathrm{Hg}$. After induction of anesthesia, the patient was monitored with arterial line, EDCO probe and tonometer probe. In the present study, arterial hypotension was defined as a decrease in MAP equal to or over $20 \%$ of the usual MAP, which was defined as the MAP measured the day before surgery. 
After induction of general anesthesia and before starting surgical procedure, anesthesia was maintained using target controlled infusions of propofol and remifentanil (target concentrations 5 $\mu . m \mathrm{l}-1$ Marsh model and 5 ng.ml-1 Minto model, respectively). During this period, patients were resuscitated using fluid challenge of saline associated or not with boluses of ephedrine, while hemodynamic monitoring was placed into the patients. After this initial phase, according to our standard of care of our institution, intra-operative episodes of hypotension were treated by a vasoconstrictor bolus of $50 \mu \mathrm{g}$ of PE ${ }^{11,31}$ or $5 \mu \mathrm{g}$ of NA based on physician's choice. With the applied concentrations of PE and NA an equivalent effect on the MAP was expected from an equal infusion rate. ${ }^{17,32}$

Vasopressors were administrated via a peripheral venous catheter. Adverse effects after vasopressors boluses, as severe hypertension (MAP>120 mmHg) or extra-vascular infusion, were collected.

As each patient may have received several boluses of the two vasoconstrictors, only boluses administered to treat general anesthesia-induced arterial hypotension with following characteristics were analyzed: (1) stable hemodynamic state with no acute change of MAP or CO 1 minute before bolus (2) no clear evidence of hypovolemia or acute hemorrhage, (3) no concomitant rapid fluid administration, (4) no change in respiratory or ventilator parameters or anesthesia infusion rate 3 minutes before or during bolus and (4) in case of multiple boluses in a short interval were administrated, we analyzed only the first bolus if the delay between the first and the second boluses was less than 5 minutes to try to eliminate the confounding factors such as synergism between the drugs and repetitive boluses.

\section{Statistical analysis}


The data are expressed as the median (interquartile range [IQR]) or a count and percentage. Patients' characteristics were compared between the Low and High CV risk groups using Wilcoxon test (for continuous variables) or using Fisher's exact test (for categorical variables). We also considered a predefined subgroup including only boluses, which succeed to correct hypotension within the predefined target-zone (increase from 10 to $30 \%$ ). Comparisons were then conducted between PE and NA using Student's t tests that were weighted by the number of measures obtained for each subject. Accordingly, for each individual, we summarized separately the effect of each drug by averaging the variation induced by the boluses. As the number of boluses varied from a patient to another, we used weighted methods to compare those average values, the weights were the number of measure per subject. A two-sided p value of 0.05 was considered significant. All statistical analyses were performed using R statistical software (The 'R' Foundation for Statistical Computing, Vienna, Austria). 


\section{Results:}

Seventy-seven patients were screened between February and July 2013. Thirty patients were excluded from the analysis due to the quality of the Doppler or tonometry recordings $(n=28)$, or the absence of vasopressor administration $(n=2)$. Finally, 47 patients were analyzed.

The characteristics of the patients are described in Table 1. Seventy percent of the patients were ASA $>1$. The most prevalent comorbidities were active smoking, dyslipidemia and a history of treated hypertension. Twenty-two patients were classified as High CV risk patients, whereas 25 were classified as Low CV risk patients. The neurosurgical procedures consisted of resection of cerebral tumors in $31(66 \%)$ patients, resection of intra-cranial aneurysm in $4(9 \%)$ patients, and spine surgery in supine position in $5(11 \%)$ patients. During the initial resuscitation, 33 (70\%) of the subjects received EPH with a median (interquartile range) dose of $9 \mathrm{mg}$ [6 - 12].

After the initial phase, all patients received a total of 269 bolus injections of vasopressors, including 149 of NA and 120 of PE. Forty three (91\%) patients received both NA and PE, while 2 patients received NA only and two PE only. No adverse effects, such as severe hypertension or extra-vascular infusion, were noted for any vasopressors during the study. Table 2 shows that boluses of $5 \mathrm{mcg}$ of NA increased MAP by an average of $22 \pm 8 \%$, which was a slightly lower than changes of MAP with boluses of $50 \mu \mathrm{g}$ of PE $(+25 \pm 7 \%$; $=0.002)$. A more significant decrease in SV was observed with PE compared to NA $(-18 \pm 9 \%$ vs $-14 \pm 7 \%$, p<0.001). PEinduced decrease in SV was associated with a gradual increase in Aix and a decrease in Ctot, which was more pronounced for PE than for NA $(+10 \pm 8 \%$ vs $+6 \pm 6 \%, \mathrm{p}<0.0001$ for $\Delta$ Aix; and $35 \pm 9 \%$ vs $-29 \pm 10 \%, \mathrm{p}<0.001$ for $\Delta$ Ctot, respectively). Seventy-two percent of boluses corrected hypotension within the predefined target-zone (increase from 10 to $30 \%$ ). When considering only those boluses (Table 2 and Figure 1), we observed for the same increase in MAP a more 
prominent decrease in $\mathrm{CO}$ and in Ctot associated with a more prominent increase in Aix when using PE compared to NA.

When the differential effect of NA and PE was tested according to the CV risk factors, our results confirmed that NA produced smaller decreases in SV, HR and CO than PE for the same increase in MAP in both Low and High CV risk patients (Table 3). In addition, arterial stiffness, as evaluated by central PP and Ctot, seemed significantly more altered by PE compared to NA in High CV risk patients, whereas for Low CV risk patients' arterial stiffness changes were similar between the two drugs (Table 3). Figure 2 of ESM depicted two actual cases illustrating the difference between a low and a high CV risk patients.

We also duplicated all comparisons and performed sensitivity analyses in the subset of patients $(n=43)$ who received at least one dose of each drug. The results obtained were similar to those obtained in the entire population (Supplementary Tables). 


\section{Discussion}

Our results showed that a $5 \mu \mathrm{g}$ NA bolus administered in a peripheral venous line treated general-anesthesia-induced arterial hypotension with the following benefits: i) an adequate target of a $20 \%$ increase in MAP with no adverse effect; ii) a better effect on ventricular afterload with a lower decrease in stroke volume and arterial compliance when compared to PE, and iii) these effects were present in both High and Low CV risk patients, with probably a more pronounced beneficial effect in High risk patients. Thus, a low dose of NA could represent a highly conceivable alternative to treat general-anesthesia-induced arterial hypotension after the initial use of EPH.

The cardiovascular tolerance of EPH is due to the pharmacodynamic properties of this drug. EPH causes endogenous catecholamine release, which is responsible for the effect on vasomotricity, but it also has chronotropic and inotropic effects, which improve myocardial functioning. This explains why EPH is used in the first attempt to treat hypotensive episodes in the operation room. However, tachyphylaxis inherent to this molecule limits the cumulative dose during long procedures.

PE, which binds specifically with the arteriolar alpha 1 receptor, is responsible for intensive and isolated vasoconstriction. This might explain, as already described, the increase in left ventricular afterload and arterial stiffness, which caused the significant decreases in stroke volume and cardiac output observed in our study ${ }^{10,33}$.

Concerning NA, inotropic and chronotropic effects have been described, along with vasoactive effects at very low doses ${ }^{34,35}$. In addition, NA causes an arterial and venous vasoconstriction, thus improving venous return and cardiac preload ${ }^{36,37}$. These effects could 
explain the decreased effects of NA on cardiac afterload and cardiac output when compared to PE in our population. NA is commonly administered by continuous infusion and at higher doses during shock in the operating room or critical care unit. However, it was recently suggested that the intraoperative strategy of using a continuous low-dose infusion of NA could improve the prognosis of patients by reducing intraoperative fluid loading during major surgery ${ }^{38}$. A few studies have described the effect for bolus administration and compared to other vasopressors. Our study showed that the use of very diluted solutions of NA $(5 \mu \mathrm{g} / \mathrm{ml})$ could correct hypotension induced by general anesthesia, without complications and with less decrease of arterial compliance when compared to PE. Interestingly, in our study the increase in arterial rigidity induced by NA compared to PE seems to be lower mainly in High CV risk patients, whereas for Low CV risk patients' arterial stiffness changes were similar between the two drugs (Table 3). We believe that those observed differences between low and high CV risk patients need to be confirmed in further large studies, but, a low dose of NA could represent a highly conceivable alternative after the initial use of EPH to treat general-anesthesia-induced arterial hypotension.

Our results are consistent with those recently published by Ngan Kee et al ${ }^{15}$. In this study, 104 patients were randomized to receive continuous infusion of either NA $5 \mu \mathrm{g} / \mathrm{mL}$ or PE $100 \mu \mathrm{g} / \mathrm{mL}$ to maintain systolic blood pressure after spinal anesthesia for cesarean delivery. The authors observed that the use of NA was associated with greater heart rate and cardiac output compared with PE. As recently reported, the use of intermittent intravenous NA boluses to prevent spinal-induced hypotension was feasible and not observed to be associated with adverse outcomes ${ }^{39}$. However, the evidence of the superiority of NA in this application is still a subject of debate. Indeed, a recent randomized controlled trial study found that NA was equivalent in 
restoring MAP but inducing the same decrease in heart rate, cardiac output and even a more marked fall in tissue perfusion as compared with PE. ${ }^{17}$ These support the use of NA to treat hypotension induced by anesthesia, but further studies are warranted to assess the superiority of NA versus PE on both central and regional perfusion, and in different populations, types of surgery and volemic conditions. Combined analyses of the effects of NA and PE on central hemodynamic and regional perfusion are needed in further investigation.

\section{Limitations of the study}

The present study has several limitations. (1) This was a non randomized unblinded prospective study which did not protocolize the use of vasoconstrictors. Thus, possible selection and performance bias cannot be excluded. (2) We chose to measure stroke volume using esophageal Doppler, which is not considered the gold standard technique ${ }^{40}$. Although significant, differences in SV variations found in the study were small and close of the errors of measurement (3) A large proportion of patients were excluded for a low quality or an inability in applying tonometry. This technique is used daily to evaluate and track patients with hypertensive disease, and its use requires training. This device, although already used in ICU patients ${ }^{30}$, likely has to be adapted to detect hypotension and track the effect of vasopressors on aortic pressure. (4) Only bolus administration of vasopressors was considered in the present study, and PE and NA are commonly used by continuous perfusion. The pharmacokinetic properties are modified by the administration mode, and this could change the effects on arterial elastance and ventricular afterload. (5) Equivalent dose-effect between of $50 \mu \mathrm{g}$ of PE compared to $5 \mu \mathrm{g}$ of NA is not absolutely clear and may confound in the results because of the concentration -response curve of each drugs. However, when comparing the groups with the same increase in MAP, our results showed a favorable effect on cardiac output and arterial compliance when using NA compared to PE (6) In our study, patients were not severely preload-dependent at the time of vasopressor 
administration because of the type of surgery and the standard of our care. As previously described, this might explain the significant deleterious effects on cardiac output generated by NA, and more importantly, by $\mathrm{PE}^{41-43}$. Further studies are needed to confirm these findings in other patient populations.

\section{Conclusion}

This preliminary study suggests that a $5 \mu \mathrm{g}$ norepinephrine bolus administered in a peripheral venous line could safely treat general-anesthesia-induced arterial hypotension with a smaller decrease in stroke volume and a smaller increase in arterial stiffness when compared with phenylephrine. Thus, a low dose of norepinephrine could represent a highly conceivable alternative to treat general-anesthesia-induced arterial hypotension. 


\section{Authors' contribution}

FV, EG and AM: conception and design of research. FV, OP, ALG and JM performed experiments. EG and OP analyzed the data. EG and FV interpreted results of experiments. EG, FV and OP prepared the figures. FV, ALG, EG and AM drafted and edited the manuscript. FV, OP, ALG, JM, AM and EG approved final version of manuscript. 


\section{References}

1. van Waes JA, van Klei WA, Wijeysundera DN, van Wolfswinkel L, Lindsay TF, Beattie WS. Association between Intraoperative Hypotension and Myocardial Injury after Vascular Surgery. Anesthesiology 2016; 124: 35-44.

2. Kheterpal S, O'Reilly M, Englesbe MJ, Rosenberg AL, Shanks AM, Zhang L, Rothman ED, Campbell DA, Tremper KK. Preoperative and intraoperative predictors of cardiac adverse events after general, vascular, and urological surgery. Anesthesiology 2009; 110: 58-66.

3. Bijker JB, Persoon S, Peelen LM, Moons KG, Kalkman CJ, Kappelle LJ, van Klei WA. Intraoperative hypotension and perioperative ischemic stroke after general surgery: a nested casecontrol study. Anesthesiology 2012; 116: 658-64.

4. Ebert TJ, Kanitz DD, Kampine JP. Inhibition of sympathetic neural outflow during thiopental anesthesia in humans. Anesthesia and analgesia 1990; 71: 319-26.

5. Marty J, Gauzit R, Lefevre P, Couderc E, Farinotti R, Henzel C, Desmonts JM. Effects of diazepam and midazolam on baroreflex control of heart rate and on sympathetic activity in humans. Anesthesia and analgesia 1986; 65: 113-9.

6. Kristensen SD, Knuuti J, Saraste A, Anker S, Botker HE, Hert SD, Ford I, GonzalezJuanatey JR, Gorenek B, Heyndrickx GR, Hoeft A, Huber K, Iung B, Kjeldsen KP, Longrois D, Luscher TF, Pierard L, Pocock S, Price S, Roffi M, Sirnes PA, Sousa-Uva M, Voudris V, FunckBrentano C, Authors/Task Force M. 2014 ESC/ESA Guidelines on non-cardiac surgery: cardiovascular assessment and management: The Joint Task Force on non-cardiac surgery: cardiovascular assessment and management of the European Society of Cardiology (ESC) and the European Society of Anaesthesiology (ESA). European heart journal 2014; 35: 2383-431.

7. Hollenberg SM. Vasoactive drugs in circulatory shock. American journal of respiratory and critical care medicine 2011; 183: 847-55. 
8. Thiele RH, Nemergut EC, Lynch C, 3rd. The clinical implications of isolated alpha(1) adrenergic stimulation. Anesthesia and analgesia 2011; 113: 297-304.

9. Thiele RH, Nemergut EC, Lynch C, 3rd. The physiologic implications of isolated alpha(1) adrenergic stimulation. Anesthesia and analgesia 2011; 113: 284-96.

10. Goertz AW, Schmidt M, Seefelder C, Lindner KH, Georgieff M. The effect of phenylephrine bolus administration on left ventricular function during isoflurane-induced hypotension. Anesthesia and analgesia 1993; 77: 227-31.

11. Goertz AW, Lindner KH, Seefelder C, Schirmer U, Beyer M, Georgieff M. Effect of phenylephrine bolus administration on global left ventricular function in patients with coronary artery disease and patients with valvular aortic stenosis. Anesthesiology 1993; 78: 834-41.

12. Mets B. Should Norepinephrine, Rather than Phenylephrine, Be Considered the Primary Vasopressor in Anesthetic Practice? Anesthesia and analgesia 2016; 122: 1707-14.

13. Wuethrich PY, Burkhard FC, Thalmann GN, Stueber F, Studer UE. Restrictive deferred hydration combined with preemptive norepinephrine infusion during radical cystectomy reduces postoperative complications and hospitalization time: a randomized clinical trial. Anesthesiology 2014; 120: 365-77.

14. Wuethrich PY, Studer UE, Thalmann GN, Burkhard FC. Intraoperative continuous norepinephrine infusion combined with restrictive deferred hydration significantly reduces the need for blood transfusion in patients undergoing open radical cystectomy: results of a prospective randomised trial. European urology 2014; 66: 352-60.

15. Ngan Kee WD, Lee SW, Ng FF, Tan PE, Khaw KS. Randomized double-blinded comparison of norepinephrine and phenylephrine for maintenance of blood pressure during spinal anesthesia for cesarean delivery. Anesthesiology 2015; 122: 736-45. 
16. Kwak YL, Lee CS, Park YH, Hong YW. The effect of phenylephrine and norepinephrine in patients with chronic pulmonary hypertension*. Anaesthesia 2002; 57: 9-14.

17. Poterman M, Vos JJ, Vereecke HE, Struys MM, Vanoverschelde H, Scheeren TW, Kalmar AF. Differential effects of phenylephrine and norepinephrine on peripheral tissue oxygenation during general anaesthesia: A randomised controlled trial. Eur J Anaesthesiol 2015; 32: $571-80$.

18. Ky B, French B, May Khan A, Plappert T, Wang A, Chirinos JA, Fang JC, Sweitzer NK, Borlaug BA, Kass DA, St John Sutton M, Cappola TP. Ventricular-arterial coupling, remodeling, and prognosis in chronic heart failure. Journal of the American College of Cardiology 2013; 62: 1165-72.

19. Reference Values for Arterial Stiffness C. Determinants of pulse wave velocity in healthy people and in the presence of cardiovascular risk factors: 'establishing normal and reference values'. European heart journal 2010; 31: 2338-50.

20. Williams B, Lacy PS, Thom SM, Cruickshank K, Stanton A, Collier D, Hughes AD, Thurston H, O'Rourke M, Investigators C, Anglo-Scandinavian Cardiac Outcomes Trial I, Committee CS, Writing C. Differential impact of blood pressure-lowering drugs on central aortic pressure and clinical outcomes: principal results of the Conduit Artery Function Evaluation (CAFE) study. Circulation 2006; 113: 1213-25.

21. Sharir T, Marmor A, Ting CT, Chen JW, Liu CP, Chang MS, Yin FC, Kass DA. Validation of a method for noninvasive measurement of central arterial pressure. Hypertension 1993; 21: 74-82.

22. Chen CH, Ting CT, Nussbacher A, Nevo E, Kass DA, Pak P, Wang SP, Chang MS, Yin FC. Validation of carotid artery tonometry as a means of estimating augmentation index of ascending aortic pressure. Hypertension 1996; 27: 168-75. 
23. Hirata K, Kawakami M, O'Rourke MF. Pulse wave analysis and pulse wave velocity: a review of blood pressure interpretation 100 years after Korotkov. Circulation journal : official journal of the Japanese Circulation Society 2006; 70: 1231-9.

24. McEniery CM, Yasmin, Hall IR, Qasem A, Wilkinson IB, Cockcroft JR, Investigators A. Normal vascular aging: differential effects on wave reflection and aortic pulse wave velocity: the Anglo-Cardiff Collaborative Trial (ACCT). Journal of the American College of Cardiology 2005; 46: $1753-60$.

25. Rowe JW. Clinical consequences of age-related impairments in vascular compliance. The American journal of cardiology 1987; 60: 68G-71G.

26. London GM, Guerin AP, Pannier B, Marchais SJ, Stimpel M. Influence of sex on arterial hemodynamics and blood pressure. Role of body height. Hypertension 1995; 26: 514-9.

27. Thompson J, Khalil RA. Gender differences in the regulation of vascular tone. Clinical and experimental pharmacology \& physiology 2003; 30: 1-15.

28. Kelly RP, Gibbs HH, O'Rourke MF, Daley JE, Mang K, Morgan JJ, Avolio AP. Nitroglycerin has more favourable effects on left ventricular afterload than apparent from measurement of pressure in a peripheral artery. European heart journal 1990; 11: 138-44.

29. Chen CH, Nevo E, Fetics B, Pak PH, Yin FC, Maughan WL, Kass DA. Estimation of central aortic pressure waveform by mathematical transformation of radial tonometry pressure. Validation of generalized transfer function. Circulation 1997; 95: 1827-36.

30. Lamia B, Teboul JL, Monnet X, Osman D, Maizel J, Richard C, Chemla D. Contribution of arterial stiffness and stroke volume to peripheral pulse pressure in ICU patients: an arterial tonometry study. Intensive Care Med 2007; 33: 1931-7. 
31. Ducrocq N, Kimmoun A, Furmaniuk A, Hekalo Z, Maskali F, Poussier S, Marie PY, Levy B. Comparison of equipressor doses of norepinephrine, epinephrine, and phenylephrine on septic myocardial dysfunction. Anesthesiology 2012; 116: 1083-91.

32. Barash P, Cullen B, Stoelting R. Clinical anesthesia. Philadelphia: Lippincott Williams \& Wilkins, 2006.

33. Goertz AW, Seeling W, Heinrich H, Lindner KH, Rockemann MG, Georgieff M. Effect of phenylephrine bolus administration on left ventricular function during high thoracic and lumbar epidural anesthesia combined with general anesthesia. Anesthesia and analgesia 1993; 76 : 541-5.

34. Fawaz G, Tutunji B. The effect of adrenaline and noradrenaline on the metabolism and performance of the isolated dog heart. British journal of pharmacology and chemotherapy 1960; 15: 389-95.

35. Goldberg LI, Cotten MD, Darby TD, Howell EV. Comparative heart contractile force effects of equipressor doses of several sympathomimetic amines. The Journal of pharmacology and experimental therapeutics 1953; 108: 177-85.

36. Monnet X, Jabot J, Maizel J, Richard C, Teboul JL. Norepinephrine increases cardiac preload and reduces preload dependency assessed by passive leg raising in septic shock patients. Critical care medicine 2011; 39: 689-94.

37. Persichini R, Silva S, Teboul JL, Jozwiak M, Chemla D, Richard C, Monnet X. Effects of norepinephrine on mean systemic pressure and venous return in human septic shock. Critical care medicine 2012; 40: 3146-53.

38. Chappell D, Jacob M, Hofmann-Kiefer K, Conzen P, Rehm M. A rational approach to perioperative fluid management. Anesthesiology 2008; 109: 723-40. 
39. Onwochei DN, Ngan Kee WD, Fung L, Downey K, Ye XY, Carvalho JC. Norepinephrine Intermittent Intravenous Boluses to Prevent Hypotension During Spinal Anesthesia for Cesarean Delivery: A Sequential Allocation Dose-Finding Study. Anesthesia and analgesia 2017.

40. DiCorte CJ, Latham P, Greilich PE, Cooley MV, Grayburn PA, Jessen ME. Esophageal Doppler monitor determinations of cardiac output and preload during cardiac operations. The Annals of thoracic surgery 2000; 69: 1782-6.

41. Caillard A, Gayat E, Tantot A, Dubreuil G, M'Bakulu E, Madadaki C, Bart F, Bresson D, Froelich S, Mebazaa A, Vallee F. Comparison of cardiac output measured by oesophageal Doppler ultrasonography or pulse pressure contour wave analysis. British journal of anaesthesia 2015; 114: 893-900.

42. Cannesson M, Jian Z, Chen G, Vu TQ, Hatib F. Effects of phenylephrine on cardiac output and venous return depend on the position of the heart on the Frank-Starling relationship. Journal of applied physiology 2012; 113: 281-9.

43. Maas JJ, Pinsky MR, de Wilde RB, de Jonge E, Jansen JR. Cardiac output response to norepinephrine in postoperative cardiac surgery patients: interpretation with venous return and cardiac function curves. Critical care medicine 2013; 41: 143-50. 
Table 1. Characteristics of the study subjects

\begin{tabular}{|c|c|c|c|c|}
\hline & $\begin{array}{c}\text { All subjects } \\
(n=47)\end{array}$ & $\begin{array}{c}\text { Low CV risk } \\
(n=25)\end{array}$ & $\begin{array}{c}\text { High CV risk } \\
(n=22)\end{array}$ & $P$ value \\
\hline \multicolumn{5}{|l|}{ Patient characteristics } \\
\hline Gender (Male/Female) & $19(40) / 28(60)$ & $6(24) / 19(76)$ & $13(59) / 9(41)$ & 0.014 \\
\hline Age (years) & $53[39-64]$ & $41[34-52]$ & $64[55-70]$ & $<0.001$ \\
\hline ASA score $(\mathrm{n}(\%))$ & & & & 0.0093 \\
\hline 1 & $14(30)$ & $12(48)$ & $2(9.1)$ & \\
\hline 2 & $25(53)$ & $10(40)$ & $15(68.2)$ & \\
\hline 3 & $7(15)$ & $3(12)$ & $4(18.2)$ & \\
\hline 4 & $1(2)$ & $0(0)$ & $1(4.5)$ & \\
\hline \multicolumn{5}{|l|}{ Comorbidities (n (\%)) } \\
\hline History of hypertension & $13(28)$ & $0(0)$ & $13(59.1)$ & $<0.001$ \\
\hline Coronary artery disease & $5(13)$ & $0(0)$ & $6(27.3)$ & 0.0052 \\
\hline Chronic obstructive pulmonary disease & $5(11)$ & $1(4)$ & $4(18.2)$ & 0.12 \\
\hline Diabetes mellitus & $5(11)$ & $1(4)$ & $4(18.2)$ & 0.12 \\
\hline Chronic renal failure & $4(9)$ & $0(0)$ & $4(18.2)$ & 0.026 \\
\hline History of stroke & $5(11)$ & $3(12)$ & $2(9.1)$ & 0.75 \\
\hline Dyslipidemia & $12(26)$ & $1(4)$ & $11(50)$ & 0.00031 \\
\hline Tobacco use & $23(50)$ & $10(40)$ & $13(59.1)$ & 0.19 \\
\hline \multicolumn{5}{|l|}{ Lee Criteria } \\
\hline 1 & $32(68)$ & $23(92)$ & $9(41)$ & $<0.01$ \\
\hline 2 & $12(26)$ & $2(8)$ & $10(45)$ & $<0.01$ \\
\hline 3 & $3(6)$ & $0(0)$ & $3(13)$ & 0.055 \\
\hline \multicolumn{5}{|l|}{ Anti-Hypertensive Treatment } \\
\hline IEC/ARA II & $8(17)$ & $0(0)$ & $8(36)$ & $<0.01$ \\
\hline Beta Blocker & $7(14)$ & $0(0)$ & $7(31)$ & $<0.01$ \\
\hline Others & $3(6)$ & $1(4)$ & $2(9)$ & 0.47 \\
\hline Duration of surgery (min) & $320[210-450]$ & $325[225-460]$ & 295 [203-443] & 0.66 \\
\hline Perioperative fluid administration (ml) & $4000[3000-4500]$ & $4000[3500-5500]$ & $3750[2875-4000]$ & 0.12 \\
\hline Perioperative bleeding (ml) & $0[0-300]$ & $200[0-350]$ & $0[0-50]$ & 0.049 \\
\hline Perioperative diuresis (ml) & $675[475-1420]$ & 925 [500-1497.5) & 525 [395-925] & 0.095 \\
\hline
\end{tabular}

Averages of hemodynamic parameters

\begin{tabular}{lcccc}
\hline MAP (mmHg) & $69+/-6$ & $69+/-5$ & $70+/-6$ & 0.06 \\
$\mathrm{CO}(\mathrm{L} / \mathrm{min})$ & $5.30+/-1.54$ & $5.86+/-1.81$ & $4.71+/-0.91$ & $<0.001$ \\
$\mathrm{SV}(\mathrm{mL})$ & $74+/-19$ & $77+/-24$ & $72+/-12$ & 0.01 \\
$\mathrm{HR}(\mathrm{bpm})$ & $72+/-12$ & $77+/-10$ & $67+/-12$ & $<0.001$ \\
Peripheral PP (mmHg) & $44+/-9$ & $44+/-8$ & $45+/-9$ & 0.59 \\
Central PP (mmHg) & $33+/-8$ & $31+/-7$ & $34+/-8$ & 0.003 \\
Aix $(\%)$ & $131+/-20$ & $127+/-20$ & $135+/-21$ & $<0.001$ \\
Ctot (mmHg/mL) & $2.43+/-0.81$ & $2.61+/-0.97$ & $2.23+/-0.55$ & $<0.001$ \\
\hline
\end{tabular}

Abbreviations: MAP, mean arterial pressure. CO, cardiac output. SV, stroke volume. HR, heart rate. PP, pulse pressure. Aix, augmentation index. Ctot, total compliance. 
Table 2. Comparison of hemodynamic variations between norepinephrine and phenylephrine in the entire population $(n=47)$.

\begin{tabular}{|c|c|c|c|c|c|c|}
\hline & \multicolumn{3}{|c|}{ All boluses } & \multicolumn{3}{|c|}{$\triangle M A P$ within $10 \%$ and $30 \%$} \\
\hline & Norepinephrine & Phenylephrine & p value & Norepinephrine & Phenylephrine & p value \\
\hline \multicolumn{7}{|l|}{ МMAP } \\
\hline - $\quad m m H g$ & $15+/-5$ & $17+/-4$ & 0.004 & $14+/-3$ & $14+/-3$ & 0.16 \\
\hline - $\%$ & $22+/-8$ & $25+/-7$ & 0.002 & $20+/-4$ & $21+/-4$ & 0.16 \\
\hline \multicolumn{7}{|l|}{$\Delta C O$} \\
\hline - $\quad L / \min$ & $-1.01+/-0.62$ & $-1.37+/-0.78$ & $<0.001$ & $-1.03+/-0.61$ & $-1.34+/-0.74$ & 0.003 \\
\hline - $\%$ & $-19+/-9$ & $-25+/-10$ & $<0.001$ & $-19+/-9$ & $-25+/-10$ & $<0.001$ \\
\hline \multicolumn{7}{|l|}{$\Delta \mathbf{S V}$} \\
\hline - $m L$ & $-11+/-7$ & $-14+/-9$ & 0.003 & $-10+/-7$ & $-13+/-8$ & 0.02 \\
\hline - $\%$ & $-14+/-7$ & $-18+/-9$ & $<0.001$ & $-14+/-7$ & $-18+/-9$ & 0.002 \\
\hline \multicolumn{7}{|l|}{$\Delta H R$} \\
\hline - $\quad b p m$ & $-4+/-4$ & $-7+/-4$ & $<0.001$ & $-4+/-4$ & $-7+/-4$ & $<0.001$ \\
\hline - $\%$ & $-5+/-5$ & $-9+/-6$ & $<0.001$ & $-6+/-5$ & $-9+/-6$ & $<0.001$ \\
\hline \multicolumn{7}{|l|}{$\Delta_{\text {central }} \mathbf{P P}$} \\
\hline - $\quad m m H g$ & $8+/-5$ & $9+/-6$ & 0.03 & $7+/-4$ & $8+/-6$ & 0.27 \\
\hline - $\%$ & $24+/-15$ & $30+/-20$ & 0.003 & $21+/-12$ & $27+/-22$ & 0.02 \\
\hline \multicolumn{7}{|l|}{$\Delta \mathbf{A i x}$} \\
\hline - $S U$ & $8+/-8$ & $13+/-11$ & $<0.001$ & $7+/-9$ & $12+/-11$ & 0.002 \\
\hline - $\%$ & $6+/-6$ & $10+/-8$ & $<0.001$ & $6+/-7$ & $9+/-8$ & 0.001 \\
\hline \multicolumn{7}{|l|}{$\Delta \mathrm{Ctot}$} \\
\hline - $\quad \mathrm{ml} / \mathrm{mmHg}$ & $-0.69+/-0.42$ & $-0.90+/-0.51$ & $<0.001$ & $-0.63+/-0.42$ & $-0.84+/-0.48$ & 0.002 \\
\hline - $\%$ & $-29+/-10$ & $-35+/-9$ & $<0.001$ & $-27+/-10$ & $-33+/-10$ & $<0.001$ \\
\hline
\end{tabular}

Abbreviations: MAP: mean arterial pressure; CO: cardiac output; SV: stroke volume; HR: heart rate. central PP: central pulse pressure. Aix: augmentation Index; Ctot: total arterial compliance $\left(\mathrm{Ctot}=\mathrm{SV} /\right.$ central $\left._{\mathrm{PP}}\right)$ 
Table 3. Comparison of bolus effect of norepinephrine and phenylephrine for low and high cardiovascular risk patients in the entire population $(n=47)$.

\begin{tabular}{|c|c|c|c|c|c|c|c|}
\hline & & Low CV & risk patients $(\mathrm{n}=$ & & High CV & cisk patients $(\mathbf{n}=$ & \\
\hline & & Norepinephrine & Phenylephrine & p value & Norepinephrine & Phenylephrine & p value \\
\hline$\Delta \mathbf{M A}$ & & & & & & & \\
\hline - & $\mathrm{mmHg}$ & $14+/-3$ & $15+/-3$ & 0.14 & $14+/-3$ & $14+/-2$ & 0.78 \\
\hline- & $\%$ & $20+/-5$ & $21+/-4$ & 0.25 & $20+/-3$ & $20+/-3$ & 0.51 \\
\hline$\Delta \mathrm{CO}$ & & & & & & & \\
\hline- & $L / \min$ & $-1.31+/-0.67$ & $-1.6+/-0.85$ & 0.07 & $-0.8+/-0.44$ & $-1.03+/-0.45$ & 0.01 \\
\hline - & $\%$ & $-23+/-8$ & $-28+/-10$ & 0.005 & $-17+/-9$ & $-21+/-8$ & 0.008 \\
\hline$\Delta \mathbf{S V}$ & & & & & & & \\
\hline - & $\mathrm{mL}$ & $-12+/-7$ & $-16+/-8$ & 0.05 & $-9+/-7$ & $-10+/-6$ & 0.3 \\
\hline- & $\%$ & $-16+/-6$ & $-21+/-8$ & 0.004 & $-12+/-7$ & $-14+/-8$ & 0.19 \\
\hline$\Delta H R$ & & & & & & & \\
\hline- & bpm & $-6+/-4$ & $-8+/-4$ & 0.009 & $-3+/-3$ & $-5+/-4$ & $<0.001$ \\
\hline- & $\%$ & $-8+/-5$ & $-11+/-6$ & 0.009 & $-4+/-4$ & $-8+/-6$ & 0.002 \\
\hline$\Delta_{\text {centr }}$ & & & & & & & \\
\hline - & $\mathrm{mmHg}$ & $6+/-4$ & $5+/-5$ & 0.70 & $8+/-4$ & $10+/-5$ & 0.009 \\
\hline- & $\%$ & $19+/-14$ & $20+/-25$ & 0.81 & $22+/-10$ & $35+/-17$ & $<0.001$ \\
\hline$\Delta \mathbf{A i x}$ & & & & & & & \\
\hline- & SU & $9+/-7$ & $12+/-8$ & 0.09 & $6+/-9$ & $12+/-14$ & 0.01 \\
\hline - & $\%$ & $7+/-6$ & $9+/-6$ & 0.05 & $5+/-7$ & $9+/-10$ & 0.02 \\
\hline$\Delta \mathrm{Cto}$ & & & & & & & \\
\hline- & $\mathrm{ml} / \mathrm{mmHg}$ & $-0.68+/-0.56$ & $-0.8+/-0.58$ & 0.32 & $-0.59+/-0.26$ & $-0.88+/-0.34$ & $<0.001$ \\
\hline - & $\%$ & $-27+/-13$ & $-32+/-10$ & 0.07 & $-28+/-7$ & $-35+/-10$ & $<0.001$ \\
\hline
\end{tabular}

Abbreviations: MAP: mean arterial pressure; CO: cardiac output; SV: stroke volume; HR: heart rate. central PP: central pulse pressure. Aix: augmentation index; Ctot: total arterial compliance $\left(\mathrm{Ctot}=\mathrm{SV} /\right.$ central $^{\mathrm{PP}}$ ) 


\section{Figures legends}

Figure 1. Bar-plot representation of relative variations in mean arterial pressure (MAP), stroke volume (SV), augmentation index (Aix) and Total Arterial Compliance (Ctot) for norepinephrine and phenylephrine bolus injection. * indicates $\mathrm{p}$ value $<0.05$. 